\title{
Controlling chaos and Neimark-Sacker bifurcation in a discrete-time predator-prey system
}

\author{
Figen Kangalgil ${ }^{* 1}$ (1), Seval Isik ${ }^{2}$ (D) \\ ${ }^{1}$ Dokuz Eylul University, Bergama Vocational High School, 35700, Izmir, Turkey \\ ${ }^{2}$ Sivas Cumhuriyet University, Faculty of Education, Department of Mathematics and Science Education, \\ 58140, Sivas, Turkey
}

\begin{abstract}
This article is about a discrete-time predator-prey model obtained by the forward Euler method. The stability of the fixed point of the model and the existence conditions of the Neimark-Sacker bifurcation are investigated. In addition, the direction of the NeimarkSacker bifurcation is given. Moreover, OGY control method is to implement to control chaos caused by the Neimark-Sacker bifurcation. Finally, Neimark-Sacker bifurcation, chaos control strategy, and asymptotic stability of the only positive fixed point are verified with the help of numerical simulations. The existence of chaotic behavior in the model is confirmed by computing of the maximum Lyapunov exponents.
\end{abstract}

Mathematics Subject Classification (2010). 39A33, 37G35, 39A30

Keywords. predator-prey model, fixed point, stability, Neimark-Sacker bifurcation, chaotic behavior, OGY feedback control method

\section{Introduction}

The dynamics relationship in population models attract great attention in both biology and mathematical biology. There are two types of the mathematical models in the theory of population dynamic models: the continuous-time models governed by differential equations, and the discrete-time models described by difference equations. There are many research papers which are related to continuous time population models and discrete-time populations models $[2,16,26,37]$. Discrete-time models are very important in application and these models are related to applied sciences such as ecology, biology, physics, engineering, etc. [4,12-14,24,34,36]. Zhang and Zou emphasized the importance of the difference equations in their studies [37]. "In natural, some predatory or parasitoid insects and their preys are univoltine and have no overlapping generations. The growth of those species displays discrete systems and can be described by difference equations. Even if some species have a long life and overlapping generations, when population quantities are relatively small, discrete models are appropriate to depict these populations. Additionally, people usually study population change year by year (month by month, or day by day) [37]". In 1976, the possible rich dynamic behaviors in a simple discrete-time models were clearly shown by May [30]. Moreover, Jing et al. [18], Liu et al. [27], and Liu

\footnotetext{
*Corresponding Author.

Email addresses: figen.kangalgil@deu.edu.tr (F. Kangalgil), skaracan@cumhuriyet.edu.tr (S. Isik)

Received: 22.02.2019; Accepted: 06.01.2020
} 
and Dongmei [28] remarked that discrete time models can exhibit more complex dynamic behavior than continuous time models. We need to investigate discrete-time population models to compare between the continuous time population models and discrete-time population models. The discretization of continuous models is an important way to obtain discrete models. Recently, many scholars have paid attention to the discrete-time population models by using the forward Euler method. Cheng et al. [3] investigated stability and bifurcation analysis of discrete-time prey-predator model with Allee effect. Atabaigi [1] studied the stability of fixed points and analyzed bifurcation phenomena of discrete predator-prey model with group defense by using the forward Euler method. He et al. [15] investigated the dynamics of a discrete-time predator-prey model of Holling-III type. Studies of the discrete-time population models which were obtained by Euler method can be seen in $[10,11,17,19-22,27,33,38]$.

Chaos theory investigates dynamic models, and complicated and nonlinear structures. This theory has several areas of application in different disciplines such as engineering, sociology, medicine, and economics. Especially biological models, due to their complex structure, are one of the most widely used applications of chaos theory. A chaotic model is a nonlinear deterministic model which represents complex and unpredictable behaviors. The chaos control problems deal with the attempt to stabilize a chaotic model to either a periodic orbit or an equilibrium. On the other hand, the Lyapunov exponent quantifies the sensitivity of the initial conditions of a dynamic system and its value is positive for the chaotic system. So it plays an important role in the chaotic systems [5-9].

A predator-prey model which is called Leslie-Gower predator prey model has been suggested in [25]. In this model the predator growth function is different from the predator predation function. The authors supposed that the predator growth function is defined by a function of the ratio of predators and their prey. A general form of the presented model with the semi-ratio dependent functional response is as follows [32]:

$$
\begin{aligned}
& \frac{d N(t)}{d t}=N(t) g(.)-P(t) h(.), \\
& \frac{d P(t)}{d t}=P(t)\left(r_{2}-\theta \frac{P(t)}{N(t)}\right),
\end{aligned}
$$

where $N(t)$ and $P(t)$ represent population densities of prey and predator at time $t$, respectively. The function $g($.$) is the per capita growth rate of the prey in the lack of predator,$ $h($.$) is the so-called predator functional response to prey, r_{2}$ is the intrinsic growth rates of predator, and $\theta$ is a measure of the food quality that the prey ensures for conversion into predator birth. The Leslie-Gower term is the expression $\theta \frac{P(t)}{N(t)}$. This term measures the loss in the predator population on account of rareness (per capita $\frac{P(t)}{N(t)}$ ) of its favorite food $N(t)$. The functions $g($.$) and h($.$) supply the classic properties. In the literature,$ the per capita growth rate of the prey in the lack of predator $g($.$) may be constant or$ a function dependent on $N(t)$ such as $g_{1}(N):=r_{1}\left(1-\frac{N(t)}{K}\right)$. A predator functional response to prey can be prey dependent i.e. $h()=.h(N(t))$ or predator dependent i.e. $h()=.h(N(t), P(t))$. Moreover, predator functional response functions have different types; for example, Holling Type-I functional response, ratio-dependent type are used in mathematical modelling.

In recent times, a few articles in literature discussed the dynamics of predator-prey model with Leslie type. Zhou et al. [39] studied the following model:

$$
\begin{aligned}
& \frac{d N(t)}{d t}=r_{1} N(t)-\varepsilon P(t) N(t), \\
& \frac{d P(t)}{d t}=P(t)\left(r_{2}-\theta \frac{P(t)}{N(t)}\right),
\end{aligned}
$$


where the constants $r_{1}, r_{2}, \varepsilon$, and $\theta$ are larger than zero. Zhou et al. first considered the stability conditions of the fixed point for the model and then by introducing so called Allee effect in different forms, they researched the impact of this effect on the dynamics of this predator-prey model [39]. In [2], Celik incorporated the term of delay $\tau$ into the model (1.2), where $\tau \geq 0$ denotes the delay time for the predator density. In this modified model, predator density is logistic with time delay and the carrying capacity proportional to prey density. In [29], Lv et al. considered a ratio-dependent predator-prey model with multiple delays where the dynamics are logistic with the carrying capacity proportional to prey population. They investigated the stability of the positive fixed point and the presence of Hopf bifurcation.

In [15], applying the forward Euler scheme to considered model, the authors investigated the model (1.2) with the following functions $g()=.g_{1}(N)$ and $h()=.\alpha N(t) /\left(N^{2}(t)+\beta^{2}\right)$. Here the predator consumes the prey according to the Holling type-III functional response. In [33], the authors considered the model (1.1) with the following functions $g()=.g_{1}(N)$ and $h()=.\beta N(t) /\left(N^{2}(t)+b\right)$ which denotes the functional response of simplified Holling type-IV. They showed that the model undergoes Flip and Neimark-Sacker bifurcation. Similarly, [1] studied a discrete-time predator-prey system of Leslie type with generalized Holling type-III functional response $\alpha N^{2}(t) /\left(a N^{2}(t)+b N(t)+1\right)$.

Motivated by the above mentioned studies, the aim of this paper is to compare the continuous time model with the discrete time model and observe some dynamical behaviors that the continuous time model does not have. Applying the forward Euler method to continuous predator-prey model (1.2), discrete-time version of model (1.2) is obtained as

$$
\begin{gathered}
N_{t+1}=N_{t}+\delta N_{t}\left(r_{1}-\varepsilon P_{t}\right), \\
P_{t+1}=P_{t}+\delta P_{t}\left(r_{2}-\theta \frac{P_{t}}{N_{t}}\right),
\end{gathered}
$$

where $\delta>0$ is the step size. Sucu [35] has studied the Flip bifurcation analysis of the model (1.3) at the fixed point. In this study, we investigate the stability and the Neimark-Sacker bifurcation analysis of the model (1.3) in $R_{+}^{2}$ and apply OGY control method for chaos control. The rest of this article is organized as follows: In Section 2, the local stability of the fixed point of the model (1.3) is discussed. In Section 3, choosing $\delta$ as the bifurcation parameter, Neimark-Sacker bifurcation analysis is studied. It is shown that the model (1.3) undergoes Neimark-Sacker bifurcation by using the bifurcation theory [23,33]. In Section 4, OGY control strategy is applied for chaos control due to occurrence of Neimark-Sacker bifurcation. Finally in Section 5, all the obtained theoretical conclusions are supported by some numerical simulations.

\section{Stability analysis and fixed points of the system}

In this section, we will determine the fixed point of the discrete-time system and examine the stability conditions of this point. To find the fixed point of the system (1.3), we assume that

$$
N_{t}=N_{t+1}=N^{*}, P_{t}=P_{t+1}=P^{*},
$$

in the system (1.3)

$$
\begin{aligned}
& N^{*}=N^{*}+\delta N^{*}\left(r_{1}-\varepsilon P^{*}\right), \\
& P^{*}=P^{*}+\delta P^{*}\left(r_{2}-\theta \frac{P^{*}}{N^{*}}\right) .
\end{aligned}
$$


A simple calculation shows that the system (1.3) has one coexistence fixed point $E^{*}=$ $\left(\frac{r_{1} \theta}{r_{2} \varepsilon}, \frac{r_{1}}{\varepsilon}\right)$. The Jacobian matrix of the system (1.3) is

$$
J=\left(\begin{array}{cc}
1+\delta r_{1}-\varepsilon P \delta & -\varepsilon \delta N \\
\frac{\theta \delta P^{2}}{N^{2}} & 1+\delta r_{2}-2 \frac{\theta \delta P}{N}
\end{array}\right) .
$$

It is well-known that the stability of the fixed point is determined by the eigenvalues of the matrix $J$. The characteristic equation of the matrix $J$ is

$$
\lambda^{2}-\operatorname{tr} J \lambda+\operatorname{det} J=0,
$$

where $\operatorname{tr} J=2+\delta\left(r_{1}+r_{2}-P\left(\varepsilon+\frac{2 \theta}{N}\right)\right)$ and

$$
\operatorname{det} J=1+\delta\left(r_{1}+r_{2}-\varepsilon P-\frac{2 \theta P}{N}\right)+\delta^{2}\left[r_{2}\left(r_{1}-\varepsilon P r_{2}\right)+\frac{\theta P}{N}\left(3 \varepsilon P-2 r_{1}\right)\right] .
$$

Definition 2.1. A fixed point $(x, y)$ is called

i) sink if $\left|\lambda_{1}\right|<1$ and $\left|\lambda_{2}\right|<1$, and it is locally asymptotically stable,

ii) source if $\left|\lambda_{1}\right|>1$ and $\left|\lambda_{2}\right|>1$, and it is locally unstable,

iii) saddle if $\left|\lambda_{1}\right|>1$ and $\left|\lambda_{2}\right|<1$ or $\left|\lambda_{1}\right|<1$ and $\left|\lambda_{2}\right|>1$,

iv) non-hyperbolic if either $\left|\lambda_{1}\right|=1$ or $\left|\lambda_{2}\right|=1$.

Lemma 2.2. $[15,17,22,34]$ Assume $F(\lambda)=\lambda^{2}+B \lambda+C$, where $B$ and $C$ are two real constants and let $F(1)>0$. Suppose $\lambda_{1}$ and $\lambda_{2}$ are two roots of $F(\lambda)=0$. Then, the following statements hold:

i) $\left|\lambda_{1}\right|<1$ and $\left|\lambda_{2}\right|<1$ if and only if $F(-1)>0$ and $C<1$,

ii) $\left|\lambda_{1}\right|>1$ and $\left|\lambda_{2}\right|>1$ if and only if $F(-1)>0$ and $C>1$,

iii) $\left|\lambda_{1}\right|>1$ and $\left|\lambda_{2}\right|<1$ if and only if $F(-1)<0$,

iv) $\lambda_{1}$ and $\lambda_{2}$ are a pair of conjugate complex roots and $\left|\lambda_{1}\right|=\left|\lambda_{2}\right|=1$ if and only if $B^{2}-4 C<0$ and $C=1$.

The Jacobian matrix of the system (2) evaluated at the coexistence fixed point $E^{*}=$ $\left(\frac{r_{1} \theta}{r_{2} \varepsilon}, \frac{r_{1}}{\varepsilon}\right)$ is given by

$$
J\left(E^{*}\right)=\left(\begin{array}{cc}
1 & -\frac{\delta \theta r_{1}}{r_{2}} \\
\frac{\delta r_{2}^{2}}{\theta} & 1-\delta r_{2}
\end{array}\right) .
$$

The Jacobian matrix of the system (2) evaluated at the coexistence fixed point $E^{*}=$ $\left(\frac{r_{1} \theta}{r_{2} \varepsilon}, \frac{r_{1}}{\varepsilon}\right)$ is given by

$$
J\left(E^{*}\right)=\left(\begin{array}{cc}
1 & -\frac{\delta \theta r_{1}}{r_{2}} \\
\frac{\delta r_{2}^{2}}{\theta} & 1-\delta r_{2}
\end{array}\right) .
$$

The characteristic equation of the matrix $J\left(E^{*}\right)$ can be written as follows:

$$
F(\lambda)=\lambda^{2}-\left(2-\delta r_{2}\right) \lambda+1-\delta r_{2}+\delta^{2} r_{1} r_{2} .
$$

From Lemma 2.2, we have

$$
F(1)=\delta^{2} r_{1} r_{2}
$$

Since $\delta>0, r_{1}>0$, and $r_{2}>0$, then $F(1)>0$. Moreover

$$
F(-1)=\delta^{2} r_{1} r_{2}-2 \delta r_{2}+4 .
$$


Let us take $\phi(\delta)=\delta^{2} r_{1} r_{2}-2 \delta r_{2}+4$. The discriminant of the function $\phi(\delta)$ is $\Delta=$ $4\left(r_{2}^{2}-4 r_{1} r_{2}\right)$.

If $r_{2}>4 r_{1}$ (case $\Delta>0$ ), the function $\phi(\delta)$ has real two roots:

$$
\delta_{1}=\frac{r_{2}-\sqrt{r_{2}^{2}-4 r_{1} r_{2}}}{r_{1} r_{2}}, \delta_{2}=\frac{r_{2}+\sqrt{r_{2}^{2}-4 r_{1} r_{2}}}{r_{1} r_{2}} .
$$

It is easily seen that since $r_{2}>4 r_{1}$, we have $\delta_{2}>\delta_{1}>0$. If $0<\delta<\delta_{1}$ and $\delta>\delta_{2}$, then $F(-1)>0$.

If $r_{2}=4 r_{1}(\Delta>0)$, the function $\phi(\delta)$ has real roots $\delta_{1}^{\prime}=\delta_{2}^{\prime}=\frac{1}{r_{1}}$. Then for any $\delta \in\left(-\infty, \frac{1}{r_{1}}\right) \cup\left(\frac{1}{r_{1}}, \infty\right)$, we have $F(-1)>0$.

If $r_{2}<4 r_{1}(\Delta<0)$, since the function $\phi(\delta)$ does not have real roots, $F(-1)>0$ for any $\delta>0$. It is clear that if $0<\delta<\frac{1}{r_{1}}$, then $C<1$. Also if $\delta>\frac{1}{r_{1}}$, then $C>1$.

If $r_{2}>4 r_{1}$ and $\delta_{1}<\delta<\delta_{2}$, then $F(-1)<0$.

In the light of this information we can give the following proposition.

Proposition 2.3. Assume that $E^{*}$ be a positive fixed point of the system (1.3). $E^{*}$ is

i) sink fixed point if one of the following conditions holds:
a) $r_{2} \geqslant 4 r_{1}$ and $0<\delta<\frac{r_{2}-\sqrt{r_{2}^{2}-4 r_{1} r_{2}}}{r_{1} r_{2}}$
b) $r_{2}<4 r_{1}$ and $0<\delta<\frac{1}{r_{1}}$.

ii) source fixed point if one of the following conditions holds:

a) $r_{2} \geqslant 4 r_{1}$ and $\delta>\frac{r_{2}+\sqrt{r_{2}^{2}-4 r_{1} r_{2}}}{r_{1} r_{2}}$

b) $r_{2}<4 r_{1}$ and $\delta>\frac{1}{r_{1}}$.

iii) saddle fixed point if the following conditions hold:

$$
r_{2} \geqslant 4 r_{1} \text { and } \frac{r_{2}-\sqrt{r_{2}^{2}-4 r_{1} r_{2}}}{r_{1} r_{2}}<\delta<\frac{r_{2}+\sqrt{r_{2}^{2}-4 r_{1} r_{2}}}{r_{1} r_{2}} .
$$

\section{Bifurcation analysis}

\subsection{Neimark-Sacker bifurcation at the fixed point $E^{*}$}

In this section, we select the parameter $\delta$ as a bifurcation parameter to investigate the Neimark-Sacker bifurcation by using the bifurcation theory and express the conditions which Neimark-Sacker bifurcation will occur in the model (1.3). Also, we evaluate the direction of the Neimark-Sacker bifurcation.

From Lemma 2.2 (iv), we can write $N S B_{E^{*}}$ as follows:

$$
N S B_{E^{*}}=\left\{r_{1}, r_{2}, \delta, \theta, \varepsilon \in \mathbb{R}_{+}: \delta=\frac{1}{r_{1}}, r_{2}<\frac{4}{\delta}\right\} .
$$

If the parameters lie in $N S B_{E^{*}}$, the eigenvalues of $J\left(E^{*}\right)$ are a pair of complex conjugate with modulus one. This means that there exist a Neimark-Sacker bifurcation at the fixed point $E^{*}$. The eigenvalues are given by

$$
\lambda, \bar{\lambda}=\frac{\left(2-\delta r_{2}\right) \pm i \sqrt{\delta r_{2}\left(4-\delta r_{2}\right)}}{2} .
$$


Let

$$
\delta=\frac{1}{r_{1}}
$$

Obviously, we have

$$
|\lambda|=|\bar{\lambda}|=1
$$

Because of $\delta \in N S B_{E^{*}}$, we get

$$
\left.\frac{d|\lambda(\delta)|}{d \delta}\right|_{\delta=\frac{1}{r_{1}}}=r_{2} \neq 0
$$

Moreover, if

$$
\operatorname{tr} J\left(E^{*}\right)=\delta r_{2}-2 \neq 0,-1 \text { namely } r_{1} \neq r_{2}, \frac{r_{2}}{2},
$$

then

$$
\lambda^{k}(\delta) \neq 1 \text { for } k=1,2,3,4,
$$

is satisfied.

Assume that $q, p \in \mathbb{C}^{2}$ are two eigenvectors of $J\left(\delta_{N S B_{E^{*}}}\right)$ and transposed matrix $J^{T}\left(\delta_{N S B_{E^{*}}}\right)$ corresponding to $\lambda$ and $\bar{\lambda}$, respectively. We have

$$
q \sim\left(\frac{\theta}{2 \delta r_{2}^{2}}\left(\delta r_{2}+i \sqrt{\delta r_{2}\left(4-\delta r_{2}\right)}\right), 1\right)^{T},
$$

and

$$
p \sim\left(-\frac{r_{2}}{2 \theta}\left(\delta r_{2}-i \sqrt{\delta r_{2}\left(4-\delta r_{2}\right)}\right), 1\right)^{T} .
$$

The normalized eigenvectors are

$$
\begin{aligned}
& q=\left(\frac{\theta}{2 \delta r_{2}^{2}}\left(\delta r_{2}+i \sqrt{\delta r_{2}\left(4-\delta r_{2}\right)}\right), 1\right)^{T}, \\
& p=\left(\frac{r_{2}^{3} \delta^{2}-4 r_{2}^{2} \delta}{2 \theta\left(4-\delta r_{2}\right)}-\frac{i \sqrt{\delta r_{2}\left(4-\delta r_{2}\right)}}{\theta\left(4-\delta r_{2}\right)}\left(\delta r_{2}^{2}-2 r_{2}\right), \frac{1}{2\left(4-\delta r_{2}\right)}\left(3+i \sqrt{\delta r_{2}\left(4-\delta r_{2}\right)}\right)\right)^{T} .
\end{aligned}
$$

Using the transformation

$$
x_{t}=N_{t}-\frac{r_{1} \theta}{r_{2} \varepsilon}, y_{t}=P_{t}-\frac{r_{1}}{\varepsilon},
$$

the fixed point $E^{*}$ is shifted to the point $(0,0)$. Using a second order Taylor expansion for the second equation valid for $\left|x_{t}\right|<\frac{r_{1} \theta}{r_{2} \varepsilon}$ and $\left|y_{t}\right|<\frac{r_{1}}{\varepsilon}$, system (2) has the form (3.12):

$$
\left\{\begin{array}{l}
x_{t+1}=x_{t}-\frac{\delta \theta r_{1}}{r_{2}} y_{t}+F_{1}\left(x_{t}, y_{t}\right), \\
y_{t+1}=\frac{\delta r_{2}^{2}}{\theta} x_{t}+\left(1-\delta r_{2}\right) y_{t}+F_{2}\left(x_{t}, y_{t}\right),
\end{array}\right.
$$

or

$$
\left(\begin{array}{c}
x_{t} \\
y_{t}
\end{array}\right) \rightarrow J\left(E^{*}\right)\left(\begin{array}{c}
x_{t} \\
y_{t}
\end{array}\right)+\left(\begin{array}{c}
F_{1}\left(x_{t}, y_{t}\right) \\
F_{2}\left(x_{t}, y_{t}\right)
\end{array}\right),
$$

where $F_{1}\left(x_{t}, y_{t}\right)=-\varepsilon \delta x_{t} y_{t}$ and $F_{2}\left(x_{t}, y_{t}\right)=\frac{\delta \varepsilon r_{2}^{2}}{r_{1} \theta} x_{t} y_{t}-\frac{\delta \varepsilon r_{2}}{r_{1}} y_{t}^{2}$.

The system (3.13) can be expressed as

$$
\left(\begin{array}{l}
x_{n+1} \\
y_{n+1}
\end{array}\right)=J\left(E^{*}\right)\left(\begin{array}{l}
x_{n} \\
y_{n}
\end{array}\right)+\frac{1}{2} B\left(x_{n}, x_{n}\right)+\frac{1}{6} C\left(x_{n}, x_{n}, x_{n}\right)+O\left(x_{n}^{4}\right),
$$


where the multilinear vector functions of $x, y, u \in \mathbb{R}^{2}$;

$$
B(x, y)=\left(\begin{array}{c}
B_{1}(x, y) \\
B_{2}(x, y)
\end{array}\right)
$$

and

are defined by

$$
C(x, y, u)=\left(\begin{array}{l}
C_{1}(x, y, u) \\
C_{2}(x, y, u)
\end{array}\right)
$$

$$
\begin{aligned}
B_{1}(x, y) & =\left.\sum_{j, k=1}^{2} \frac{\partial^{2} F_{1}}{\partial \xi_{j} \partial \xi_{k}}\right|_{\xi=0} x_{j} y_{k} \\
& =-\varepsilon \delta\left(x_{1} y_{2}+x_{2} y_{1}\right), \\
B_{2}(x, y) & =\left.\sum_{j, k=1}^{2} \frac{\partial^{2} F_{2}}{\partial \xi_{j} \partial \xi_{k}}\right|_{\xi=0} x_{j} y_{k} \\
& =\frac{\delta \varepsilon r_{2}^{2}}{r_{1} \theta}\left(x_{1} y_{2}+x_{2} y_{1}\right)-\frac{2 \delta \varepsilon r_{2}}{r_{1}} x_{2} y_{2}, \\
C_{1}(x, y, u) & =\left.\sum_{j, k, l=1}^{2} \frac{\partial^{3} F_{1}}{\partial \xi_{j} \partial \xi_{k} \partial \xi_{l}}\right|_{\xi=0} x_{j} y_{k} u_{l} \\
& =0, \\
C_{2}(x, y, u) & =\left.\sum_{j, k, l=1}^{2} \frac{\partial^{3} F_{2}}{\partial \xi_{j} \partial \xi_{k} \partial \xi_{l}}\right|_{\xi=0} x_{j} y_{k} u_{l} \\
& =0 .
\end{aligned}
$$

When $\delta$ is close to $\delta_{N S}$ and $z \in \mathbb{C}$, the vector $x \in \mathbb{R}^{2}$ can be decomposed uniquely as

$$
X=z q+\overline{z q} .
$$

It is clear that $z=<p, X>$.

The system (3.13) can be transformed for all sufficiently small $|\delta|$ into the form

$$
z \rightarrow \lambda(\delta) z+g(z, \bar{z}, \delta)
$$

where $\lambda(\delta)=(1+\varphi(\delta)) e^{i \arctan (\delta)}$ with $\varphi\left(\delta_{N S}\right)=0$ and $g(z, \bar{z}, \delta)$ is smooth complexvalued function. After Taylor expansion of $g$ with respect to $(z, \bar{z})$, we obtain

$$
g(z, \bar{z}, \delta)=\sum_{k+l \geq 2} \frac{1}{k ! l !} g_{k l}(\delta) z^{k} \bar{z}^{l}
$$

where

$$
\begin{aligned}
g_{20}\left(\delta_{N S}\right) & =<p, B(q, q)> \\
& =-\frac{\varepsilon}{r_{1}^{2} n}\left(\left(r_{1}-r_{2}\right) n+i \sqrt{r_{2} n}\left(r_{2}+5 r_{1}\right)\right), \\
g_{11}\left(\delta_{N S}\right) & =<p, B(q, \bar{q})> \\
& =\frac{\varepsilon r_{2}}{r_{1} \sqrt{n}} i, \\
g_{02}\left(\delta_{N S}\right) & =<p, B(\bar{q}, \bar{q})> \\
& =\frac{\varepsilon}{r_{1}^{2} n}\left(\left(r_{1}-r_{2}\right) n-i \sqrt{r_{2} n}\left(3 r_{1}-r_{2}\right)\right), \\
g_{21}\left(\delta_{N S}\right) & =<p, C(q, q, \bar{q})> \\
& =0 .
\end{aligned}
$$

At the above equations, we denote $n=4 r_{1}-r_{2}$. 
The direction of the Neimark-Sacker bifurcation is obtained by $\operatorname{sign}\left(a\left(\delta_{N S}\right)\right)$. The coefficient $a\left(\delta_{N S}\right)$ is calculated by

$$
\begin{aligned}
a\left(\delta_{N S}\right) & =\operatorname{Re}\left(\frac{e^{-i \arctan \left(\delta_{N S}\right)}}{2} g_{21}\right) \\
& -\operatorname{Re}\left(\frac{\left(1-2 e^{i \arctan \left(\delta_{N S}\right)}\right) e^{-2 i \arctan \left(\delta_{N S}\right)}}{2\left(1-e^{i \arctan \left(\delta_{N S}\right)}\right)} g_{20} g_{11}\right)-\frac{1}{2}\left|g_{11}\right|^{2}-\frac{1}{4}\left|g_{02}\right|^{2},
\end{aligned}
$$

where $e^{i \arctan \left(\delta_{N S}\right)}=\lambda\left(\delta_{N S}\right)$.

We state the following theorem on Neimark-Sacker bifurcation.

Theorem 3.1. If (3.6) holds, $a\left(\delta_{N S}\right) \neq 0$ and the parameter $\delta$ changes its value in small vicinity of $N S B_{E^{*}}$, then the model (1.3) passes through a Neimark-Sacker bifurcation at only fixed point $E^{*}$. Moreover if a $\left(\delta_{N S}\right)>0$ (respectively $\left.<0\right)$, then there exists a unique repelling (respectivelyattracting) invariant closed curve which bifurcates from $E^{*}$.

\section{Chaos control}

In dynamical systems, it is expected that the system be optimized with respect to some performance criterion and chaos be avoided. Controlling chaos in discrete-time systems is a topic of great interest for many researchers in recent time [5-9].

Chaos control can be obtained using various methods in discrete-time systems. To control the chaos in the system (1.3), we study feedback control strategy. First, we apply the OGY method to the system (1.3) which was first time proposed by [31]. For this, corresponding to the system (1.3) we consider the following controlled system:

$$
\begin{aligned}
N_{t+1} & =N_{t}+\delta N_{t}\left(r_{1}-\varepsilon P_{t}\right)=f\left(N_{t}, P_{t}, r_{1}\right), \\
P_{t+1} & =P_{t}+\delta P_{t}\left(r_{2}-\theta \frac{P_{t}}{N_{t}}\right)=g\left(N_{t}, P_{t}, r_{1}\right),
\end{aligned}
$$

where $r_{1}$ is taken as the controlling parameter. Furthermore, $r_{1}$ is restricted to line in some small interval $\left|r_{1}-r_{1_{0}}\right|<\mu$ with $\mu>0$, and $r_{1_{0}}$ denotes the nominal value belonging to chaotic region. We apply the stabilizing feedback control strategy in order to move the trajectory towards the desired orbit. Suppose that $\left(N^{*}, P^{*}\right)$ be unstable fixed point of the system (2) in chaotic region produced by the emergence of Neimark-Sacker bifurcation, then the system (4.1) can be approximated in the neighborhood of the unstable fixed point $\left(N^{*}, P^{*}\right)$ by the following linear map:

$$
\left[\begin{array}{c}
N_{t+1}-N^{*} \\
P_{t+1}-P^{*}
\end{array}\right] \approx A\left[\begin{array}{c}
N_{t}-N^{*} \\
P_{t}-P^{*}
\end{array}\right]+B\left[r_{1}-r_{1_{0}}\right]
$$

where

$$
A=\left[\begin{array}{ll}
\frac{\partial f\left(N^{*}, P^{*}, r_{1}\right)}{\partial N_{t}} & \frac{\partial f\left(N^{*}, P^{*}, r_{1_{0}}\right)}{\partial P_{t}} \\
\frac{\partial g\left(N^{*}, P^{*}, r_{1_{0}}\right)}{\partial N_{t}} & \frac{\partial g\left(N^{*}, P^{*}, r_{1_{0}}\right)}{\partial P_{t}}
\end{array}\right],
$$

and

$$
B=\left[\begin{array}{c}
\frac{\partial f\left(N^{*}, P^{*}, r_{1_{0}}\right)}{\partial r} \\
\frac{\partial f\left(N^{*}, P^{*}, r_{1_{0}}\right)}{\partial r}
\end{array}\right]=\left[\begin{array}{c}
\frac{\delta^{2} r_{1_{0}}}{r_{2} \varepsilon} \\
0
\end{array}\right] .
$$


On the other hand the system (4.1) is controllable provided that the following matrix

$$
\begin{aligned}
C & =[B: A B] \\
& =\left[\begin{array}{cc}
\frac{\delta^{2} r_{1_{0}}}{r_{2} \varepsilon} & \frac{\delta^{2} r_{1_{0}}}{r_{2} \varepsilon} \\
0 & \frac{\delta \theta r_{2} r_{1}}{\varepsilon}
\end{array}\right] .
\end{aligned}
$$

Since all parameters are positive, therefore rank of $C$ is 2 .

Now, we suppose that $\left[r_{1}-r_{1_{0}}\right]=-K\left[\begin{array}{c}N_{t}-N^{*} \\ P_{t}-P^{*}\end{array}\right]$, where $K=\left[\rho_{1} \rho_{2}\right]$, then the system (4.2) can be written as follows:

$$
\left[\begin{array}{c}
N_{t+1}-N^{*} \\
P_{t+1}-P^{*}
\end{array}\right] \approx[A-B K]\left[\begin{array}{c}
N_{t}-N^{*} \\
P_{t}-P^{*}
\end{array}\right]
$$

The corresponding controller can be written as,

$$
\begin{aligned}
& N_{t+1}=N_{t}+\delta N_{t}\left(\left(r_{1_{0}}-\rho_{1}\left(N_{t}-N^{*}\right)-\rho_{2}\left(P_{t}-P^{*}\right)\right)-\varepsilon P_{t}\right), \\
& P_{t+1}=P_{t}+\delta P_{t}\left(r_{2}-\theta \frac{P_{t}}{N_{t}}\right) .
\end{aligned}
$$

Furthermore, the fixed point $\left(N^{*}, P^{*}\right)$ of $(4.5)$ is locally asymptotically stable if and only if both eigenvalues of the matrix $A-B K$ lie in an open unit disk. The Jacobian matrix $A-B K$ of the controlled system (4.5) can be written as follows:

$$
A-B K=\left[\begin{array}{cc}
1-\frac{\delta^{2} \rho_{1} r_{1_{0}}}{r_{2} \varepsilon} & -\frac{\delta^{2} r_{1_{0}}}{r_{2}}\left(1+\frac{\rho_{2}}{\varepsilon}\right) \\
\frac{\theta r_{2}^{2}}{\varepsilon} & 1+r_{2}(\delta-2 \theta)
\end{array}\right] .
$$

The characteristic equation of the Jacobian matrix $A-B K$ is given by

$$
\begin{aligned}
P(\lambda) & =\lambda^{2}-\left(2+r_{2}(\delta-2 \theta)-\frac{\delta^{2} \rho_{1} r_{1_{0}}}{r_{2} \varepsilon}\right) \lambda+1+r_{2}(\delta-2 \theta) \\
& -\frac{\delta^{2} r_{2} r_{1_{0}}}{\varepsilon}\left(\frac{\rho_{1}}{r_{2}^{2}}\left(1+r_{2}(\delta-2 \theta)\right)+\theta\left(1+\frac{\rho_{2}}{\varepsilon}\right)\right) .
\end{aligned}
$$

Let $\lambda_{1}$ and $\lambda_{2}$ be the eigenvalues of characteristic equation (4.6), then

$$
\begin{gathered}
\lambda_{1}+\lambda_{2}=2+r_{2}(\delta-2 \theta)-\frac{\delta^{2} \rho_{1} r_{1_{0}}}{r_{2} \varepsilon} \\
\lambda_{1} \lambda_{2}=1+r_{2}(\delta-2 \theta)-\frac{\delta^{2} r_{2} r_{1_{0}}}{\varepsilon}\left(\frac{\rho_{1}}{r_{2}^{2}}\left(1+r_{2}(\delta-2 \theta)\right)+\theta\left(1+\frac{\rho_{2}}{\varepsilon}\right)\right)
\end{gathered}
$$

are valid.

In order to obtain the lines of marginal stability we must solve the equations $\lambda_{1}= \pm 1$ and $\lambda_{1} \lambda_{2}=1$. These restrictions make sure that $\lambda_{1}$ and $\lambda_{2}$ have absolute value less than 1. Assume that $\lambda_{1} \lambda_{2}=1$, then Eq. (4.8) implies

$$
L_{1}:=-\frac{\delta^{2} \rho_{1} r_{1_{0}}}{r_{2} \varepsilon}\left(r_{2}(\delta-2 \theta)+1\right)+\frac{\delta \theta r_{2} \rho_{2} r_{1_{0}}}{\varepsilon}+r_{2}\left(\delta-\theta\left(\delta r_{1}-2\right)\right)=0 .
$$

Moreover, we suppose that $\lambda_{1}=1$, then (4.7) and (4.8) yield,

$$
L_{2}:=-\frac{\delta^{2} \rho_{1} r_{1_{0}}}{\varepsilon}(\delta-2 \theta)+\frac{\delta \theta r_{2} \rho_{2} r_{1_{0}}}{\varepsilon}+\delta \theta r_{2} r_{1_{0}}=0 .
$$

Finally, taking $\lambda_{1}=-1$ and using the equations (4.7) and (4.8) we get

$$
L_{3}:=-\frac{\delta^{2} \rho_{1} r_{1_{0}}}{r_{2} \varepsilon}\left(r_{2}(\delta-2 \theta)+2\right)+\frac{\delta \theta r_{2} \rho_{2} r_{1_{0}}}{\varepsilon}+r_{2}\left(2(\delta-2 \theta)+\delta \theta r_{1_{0}}\right)+4=0 .
$$

Then, stable eigenvalues lie within the triangular region in $\rho_{1} \rho_{2}$ plane bounded by the straight lines $L_{1}, L_{2}, L_{3}$ for particular parametric values. 


\section{Numerical simulations}

Example 5.1. For the parameter values $r_{1}=4, r_{2}=14, \delta=0.2, \theta=50, \varepsilon=0.4$, and the initial condition $\left(N_{0}, P_{0}\right)=(34,9.6)$, the positive fixed point of the system (2) is obtained as $\left(N^{*}, P^{*}\right)=(35.71428571,10)$. From Proposition 2.3 (i.b), the fixed point $\left(N^{*}, P^{*}\right)$ of the system (1.3) is locally asymptotically stable (See Figure 1).
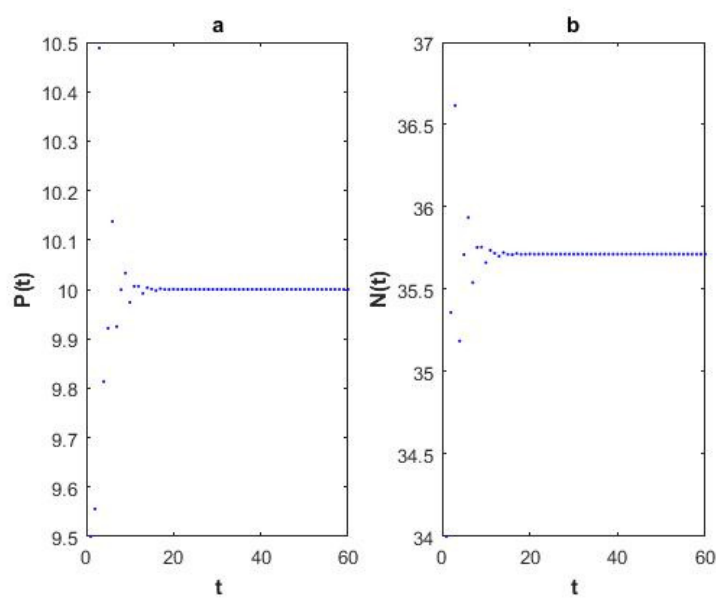

Figure 1. A stable fixed point for the system (1.3) for $r_{1}=4, r_{2}=14, \delta=$ $0.2, \theta=50, \varepsilon=0.4$, and the initial condition $\left(N_{0}, P_{0}\right)=(34,9.6)$.

Example 5.2. In this example, we give the bifurcation diagrams and phase portraits for the system (1.3) around the fixed point $\left(N^{*}, P^{*}\right)$ to confirm the above analytic results and show some interesting complex dynamical behaviors in system (1.3). We will choose $\delta$ as bifurcation parameter. We obtain the Neimark-Sacker bifurcation point as $\delta_{N S}=0.25$. For the parameter values $r_{1}=4, r_{2}=14, \theta=50, \varepsilon=0.4, \delta_{N S}=0.25$, the positive fixed point $\left(N^{*}, P^{*}\right)$ of the model $(1.3)$ is evaluated as $\left(N^{*}, P^{*}\right)=(35.71428571,10)$. Because of computing the coefficients of normal form, fixed point $\left(N^{*}, P^{*}\right)$ can be transformed into origin by change of variables as follows:

$$
\begin{aligned}
& x=N-35.71428571, \\
& y=P-10 .
\end{aligned}
$$

So, the system (1.3) converts to

$$
\begin{aligned}
& x_{n+1}=x_{n}+35.71428571-0.1\left(x_{n}+35.71428571\right) y_{n}, \\
& y_{n+1}=y_{n}+10+\frac{\left(y_{n}+10\right)\left(14-\frac{50\left(y_{n}+10\right)}{x_{n}+35.71428571}\right)}{4} .
\end{aligned}
$$

Using the above parameters, we get

$$
J\left(N^{*}, P^{*}\right)=\left[\begin{array}{cc}
1 & -3.571428571 \\
0.9800000002 & -2.5
\end{array}\right] .
$$

The eigenvalues are obtained as

$$
\begin{aligned}
& \lambda_{1}=-0.7500000000+0.6614378280 i, \\
& \lambda_{2}=-0.7500000000-0.6614378280 i .
\end{aligned}
$$

Let $q, p \in C^{2}$ be complex eigenvectors corresponding to $\lambda_{1}, \lambda_{2}$, respectively,

$$
q \sim(-0.6749365589+1.785714285 i, i)^{T},
$$


and

$$
p \sim\left(-0.7408103671-3.108773710 .10^{\wedge}(-10) i, 1.322875655+0.5000000004 i\right)^{T} .
$$

To obtain the normalization $\langle p, q\rangle=1$, we can take normalized vectors as

$$
q=(-0.6749365589+1.785714285 i, i)^{T}
$$

and

$$
p=\left(-0.7408103671-0.310877371010^{-9} i,-1.322875655+0.5000000004 i\right)^{T} .
$$

By using the formula (3.18) the coefficients of the normal of the system (4.1) can be computed as follows:

$$
\begin{aligned}
& g_{20}(\delta)=0.2753741158-0.1000000000 i \\
& g_{11}(\delta)=-0.05000000002-0.1376870578 i \\
& g_{02}(\delta)=0.1403868039-0.2571428570 i \\
& g_{21}(\delta)=0 .
\end{aligned}
$$

From (3.19), the critical part is obtained as $a\left(\delta_{N S}\right)=0.01023799478>0$. Therefore, a subcritical Neimark-Sacker bifurcation occurs at $\delta_{N S}=0.25$ and it shows correctness of Theorem 3.1. The bifurcation diagram and the phase portrait of the system (1.3) are shown in Figure 2 and Figure 3 where the initial point is $\left(N_{0}, P_{0}\right)=(34,9.5)$.

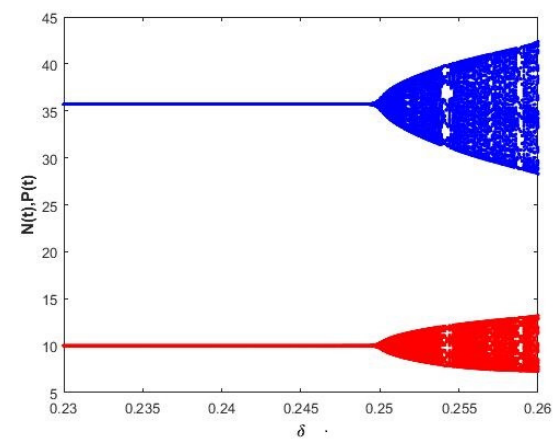

(a)

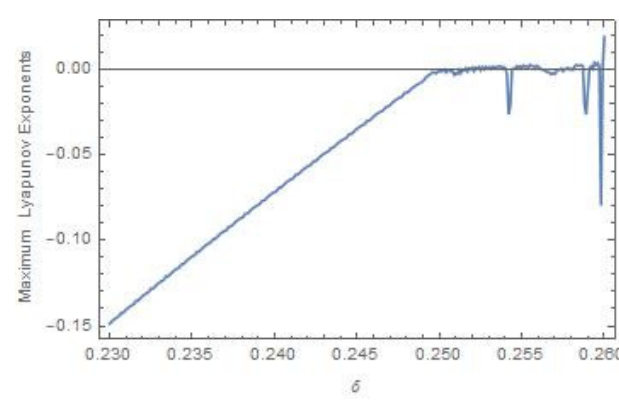

(b)

Figure 2. Bifurcation diagram and MLE for the system (1.3) for values of $r_{1}=4, r_{2}=14, \delta=(0.23,0.26),. \theta=50, \varepsilon=0.4$, and the initial condition

$$
\left(N_{0}, P_{0}\right)=(34,9.5) \text {. }
$$

From Figure 2, we observe that the fixed point $\left(N^{*}, P^{*}\right)=(35.71428571,10)$ of the system (1.3) is stable for $\delta<0.25$ and loses its stability at $\delta=0.25$, and invariant close curves appear from the positive fixed point when the parameter $\delta$ exceeds 0.25 . The phase portraits of the bifurcation diagrams in Figure 2 for different values of $\delta$ are displayed in Figure 3, which clearly depicts the process of how a smooth invariant circle bifurcates from the stable fixed point $\left(N^{*}, P^{*}\right)=(35.71428571,10)$. When $\delta$ exceeds 0.25 , then the fixed point $\left(N^{*}, P^{*}\right)=(35.71428571,10)$ is unstable and meanwhile a repelling invariant closed curve bifurcates from the positive fixed point. 

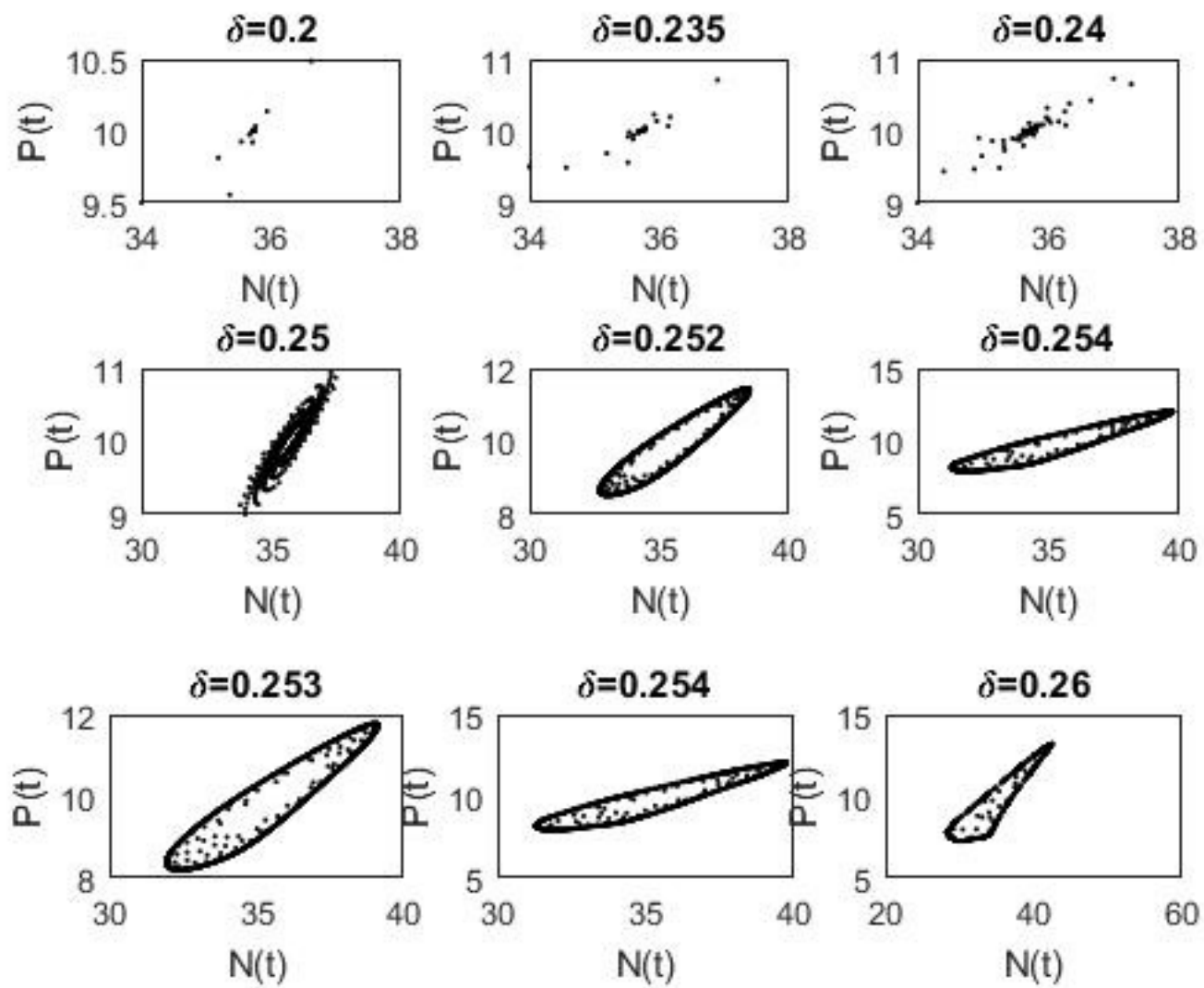

Figure 3.Phase portraits of the system (1.3) for different values of $\delta$.

Example 5.3. Let $r_{1}=3.7037037037, r_{2}=12, \delta=0.27, \theta=50, \varepsilon=0.4, r_{1} \in[3.6,4.5]$, and $\left(N_{0}, P_{0}\right)=(34,8.1)$, then the system (1.3) undergoes Neimark-Sacker bifurcation. For these parametric values, bifurcation diagram, and corresponding maximum Lyapunov exponents are plotted in Figure 4. The system (1.3) has a unique positive equilibrium point $\left(N^{*}, P^{*}\right)=(38.58024692,9.259259260)$ at these parametric values and characteristic values of the Jacobian matrix evaluated at this fixed point are given by

$$
\left|\lambda_{1,2}\right|=|-0.62 \pm 0.7846018095 I|=1 .
$$

In order to discuss the OGY feedback control method for the system (1.3), we take $r_{1_{0}}=$ 3.725.Then corresponding controlled system is given by

$$
\begin{gathered}
N_{t+1}=N_{t}+0.27 N_{t}\left(\left(3.725-\rho_{1}(N-38.80208332)-\rho_{2}(P-9.3125)\right)-\varepsilon P_{t}\right), \\
\left.P_{t+1}=P_{t}+0.27 P_{t}\left(12-50 \frac{P_{t}}{N_{t}}\right),\right],
\end{gathered}
$$

when $K=\left[\begin{array}{ll}\rho_{1} & \rho_{2}\end{array}\right]$ and $\left(N^{*}, P^{*}\right)=(38.58024692,9.259259260)$ is unstable fixed point of the system (1.3). We have

$$
\begin{aligned}
& A=\left[\begin{array}{cc}
1 & -4.190625 \\
0.7776 & -2.24
\end{array}\right], \\
& B=\left[\begin{array}{c}
10.47656250 \\
0
\end{array}\right],
\end{aligned}
$$


and

$$
\begin{aligned}
C & =[B: A B] \\
& =\left[\begin{array}{cc}
10.47656250 & 10.47656250 \\
0 & 8.146575000
\end{array}\right] .
\end{aligned}
$$

Then it is easy to check that the rank of $C$ matrix is 2. Therefore the system (5.2) is controllable. Then, the Jacobian matrix $A-B K$ of the controlled system (5.2) is given by

$$
A-B K=\left[\begin{array}{cc}
1-10.47656250 \rho_{1} & -4.190625-10.47656250 \rho_{2} \\
0.7776 & -2.24
\end{array}\right] .
$$

Moreover, the lines $L_{1}, L_{2}$, and $L_{3}$ for marginal stability are given by

$$
\begin{aligned}
L_{1}= & 0.01863+23.4675 \rho_{1}+8.146575 \rho_{1}=0, \\
L_{2}= & 3.25863+33.94406250 \rho_{1}+8.146575 \rho_{2}=0, \\
& \text { and } \\
L_{3}= & 0.77863+12.99093750 \rho_{1}+8.146575 \rho_{2}=0 .
\end{aligned}
$$

Then, the stable triangular region bounded by marginal lines $L_{1}, L_{2}$, and $L_{3}$ for the controlled system (5.2) is shown in Figure 5.

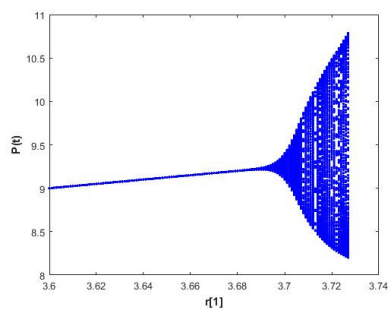

(a)

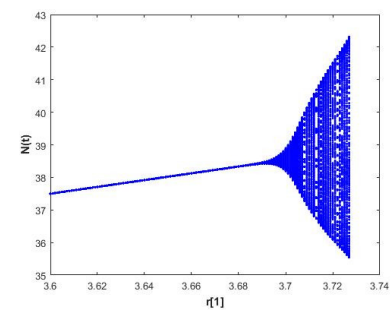

(b)

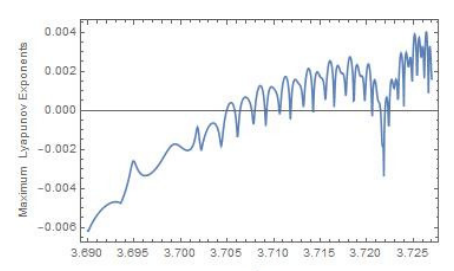

(c)

Figure 4. Bifurcation diagrams and MLE for the system (1.3) for values of $r_{2}=12$ $\delta=0.27, \theta=50, \varepsilon=0.4, r_{1}=(3.6,4.5)$, and the initial condition $\left(N_{0}, P_{0}\right)=(34,8.1)$. (a) Bifurcation diagram for $P_{t}$ (b) Bifurcation diagram for $N_{t}$ (c) Maximum Lyapunov exponents. 


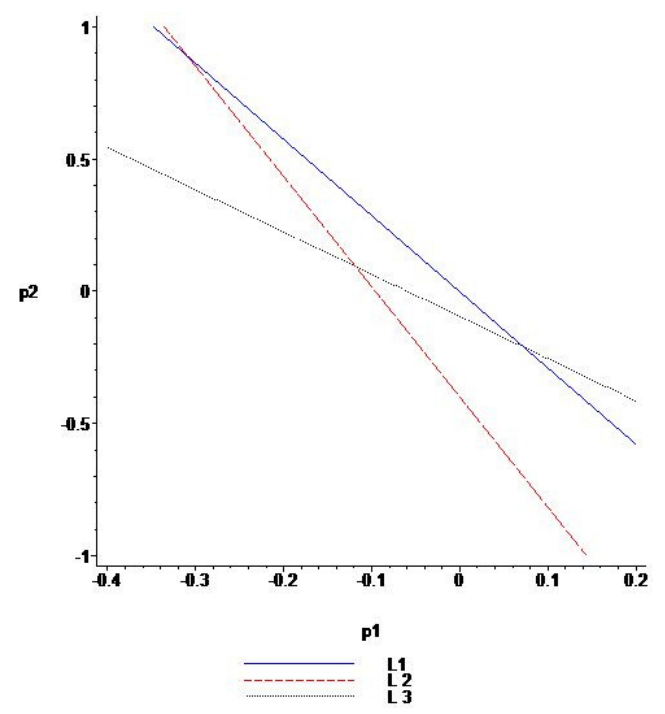

Figure 5.Triangular stability region bounded by $L_{1}, L_{2}$ and $L_{3}$ for the controlled system (5.2) .

\section{Discussions}

Previous studies have showed that discrete-time population models play an important role in mathematical biology $[4,12-14,24,34,36]$. So, we investigate the dynamical behaviors of a discrete predator-prey model. We obtain the stability conditions of the coexistence fixed point. By using bifurcation theory $[12,24,36]$ we show that the model (1.3) undergoes Neimark-Sacker bifurcation. Further, we present some numerical simulations by using MATLAB program to verify the theoretical results. We display that when the bifurcation parameter $\delta$ passes a crucial bifurcation value, the stability of the coexistence fixed point of the model (1.3) changes from stable to unstable and Neimark-Sacker bifurcation occurs at this critical value. Moreover, the model (1.3) displays the complex dynamics for different parameter values in certain regions. Therefore, we can assert that the parameter $\delta$ has a powerful effect on the stability of the model (1.3).

Model (1.2) and model (1.3), which is the discrete version of model (1.2), have both same and different dynamic properties. For example, the fixed point of model (1.2) and model (1.3) is the same. In addition, in study [2], the periodic solution occurs as a result of Hopf bifurcation that is continuous case of Neimark-Sacker bifurcation in model (1.2) at the delay parameter. However, in model (1.3) is occurred both the Neimark Sacker bifurcation and the Flip bifurcation [35]. Under the influence of the Neimark-Sacker bifurcation dynamically unstable invariant closed curves are produced. The positive sign of the maximum Lyapunov exponents in Figure 2(b) confirms the existences of the chaos and period window as parameter $\delta$ varying. In the context of biology, model (1.3) can be viewed as a predator-prey system interaction. In terms of the latter, the existence of a Neimark-Sacker bifurcation in model (1.3) implies that both the prey and predator populations can oscillate around some mean values, and these oscillations will continue indefinitely under suitable conditions. These results show far richer dynamics of the discrete model compared to the continuous model.

On the other hand, the Neimark-Sacker bifurcation is successfully controlled with OGY control method. From our numerical research, it is clear that OGY method based on feedback control strategy can restore the stability. This controlling strategy is effective 
in order to improve or entirely disappear the chaos due to occurrence of Neimark-Sacker bifurcation.

\section{References}

[1] A. Atabaigi, Multiple bifurcations and dynamics of a discrete-time predator-prey system with group defense and non-monotonic functional response, Differ. Equ. Dyn. Syst. 28, 107-132, 2020.

[2] C. Celik, The stability and Hopf bifurcation for a predator-prey system with time delay, Chaos Solitons Fractals, 37, 87-99, 2008.

[3] L. Cheng and $\mathrm{H}$. Cao, Bifurcation analysis of a discrete-time ratio-dependent predatorprey model with Allee Effect, Commun. Nonlinear Sci. Numer. Simul. 38, 288-302, 2016.

[4] M. Danca, S. Condreanu and B. Bako, Detailed analysis of a nonlinear prey-predator model, J. Biol. Phys. 23 (1), 11-20, 1997.

[5] Q. Din, A Novel chaos control strategy for discrete-time brusselator models, J. Math. Chem. 56, 3045-3075, 2018.

[6] Q. Din, Bifurcation analysis and chaos control in discrete-time glycolysis Models, J. Math. Chem. 56 (3), 904-931, 2018.

[7] Q. Din, Bifurcation analysis and chaos control in a second-order rational difference equation, Int. J. Nonlinear Sci. Numer. Simul. 19 (1), 53-68, 2018.

[8] Q. Din, Stability, bifurcation analysis and chaos control for a predator-prey system, J. Vib. Control 25 (3), 612-626, 2019.

[9] Q. Din and M. Hussain, Controlling chaos and Neimark-Sacker bifurcation in a hostparasitoid model, Asian J. Control, 21 (4), 1-14, 2019.

[10] W. Du, J. Zhang, S. Qin and J.Yu, Bifurcation analysis in a discrete SIR epidemic model with the saturated contact rate and vertical transmission, J. Nonlinear Sci. Appl. 9, 4976-4989, 2016.

[11] E.M. Elabbasy, A.A. Elsadany and Y.Zhang, Bifurcation analysis and chaos in a discrete reduced Lorenz system, Appl. Math. Comput. 228, 184-194, 2014.

[12] S.N. Elaydi, An Introduction to Difference Equations, Springer-Verlag, New York, NY, USA, 1996.

[13] A. Gkana and L. Zachilas, Incorporating prey refuge in a prey-predator model with a Holling Type I functional response: random dynamics and population outbreaks, J. Biol. Phys. 39 (4), 587-606, 2013.

[14] A. Gkana and L. Zachilas, Non-overlapping generation species: Complex PreyPredator Interactions, Int. J. Nonlinear Sci. Numer. Simul. 16 (5), 207-219, 2015.

[15] Z. He and X. Lai, Bifurcation and Chaotic Behaviour of a Discete Time Predator-Prey System, Nonlinear Anal. Real World Appl. 12 (1), 403-417, 2011.

[16] Z.M. He and B.O. Li, Complex dynamic behavior of a discrete-time predator-prey system of Holling-III Type, Adv. Difference Equ. 2014, Art. No. 180, 2014.

[17] Z.Hu, Z.Teng and L. Zhang, Stability and bifurcation analysis of a discrete predatorprey model with nonmonotonic functional response, Nonlinear Anal. Real World Appl. 12, 2356-2377, 2011.

[18] Z. Jing and Y. Jianping, Bifurcation and chaos in discrete-time predatorprey system, Chaos Solitons Fractals 27 (1), 259-277, 2006.

[19] S. Kartal, Dynamics of a plant-herbivore model with differential-difference equations, Cogent Math. 3 (1), 1136198, 2016.

[20] S. Kartal, Flip and Neimark-Sacker bifurcation in a differential equation with piecewise constant arguments model, J. Difference Equ. Appl. 23, 763-778, 2017.

[21] S.Kartal and F. Gurcan, Global behaviour of a predator-prey like model with piecewise constant arguments, J. Biol. Dyn. 9 (1), 157-171, 2015. 
[22] A.Q. Khan, Neimark-Sacker bifurcation of a two-dimensional discrete-time predatorprey model, SpringerPlus 5 (1), Art. No. 126, 2016.

[23] A.Q. Khan, Stability and Neimark-Sacker bifurcation of a ratio-dependence predatorprey model, Math. Methods Appl. Sci. 40, 4109-4119, 2017.

[24] Y. Kuznetsov, Elements of Applied Bifurcation Theory, Springer-Verlag, New York, NY, USA, 2nd edition, 1998.

[25] P.H. Leslie and J.C Gower, The properties of a stochastic model for the predator-prey type of interaction between two species, Biometrika, 47, 219-234, 1960.

[26] S. Li and W. Zhang, Bifurcations of a discrete prey-predator model with Holling type II functional response, Discrete Contin. Dyn. Syst. Ser. B. 14, 159-176, 2010.

[27] J. Liu, P. Baoyang and Z. .Tailei, Effect of discretization on dynamical behavior of SEIR and SIR models with nonlinear incidence, Appl. Math. Lett. 39, 60-66, 2015.

[28] X. Liu and X. Dongmei, Complex dynamic behaviors of a discrete-time predatorprey system, Chaos Solitons Fractals, 32 (1), 80-94, 2007.

[29] D. Lv, W. Zhang and Y. Tang, Bifurcation analysis of a ratio-dependent predator-prey system with multipla delays, J. Nonlinear Sci. Appl. 9, 3479-3490, 2016.

[30] R.M. May, Simple mathematical models with very complicated dynamics, Nature, 261, 459-467, 1976.

[31] E. Ott, C. Grebogi and J.A. Yorke, Controlling chaos, Phys. Rev. Lett. 64 (11), 1196-1199, 1990.

[32] P.J. Pal and P.K. Mandal, Bifurcation Analysis of a Modified Leslie-Gower PredatorPrey Model with Beddington-De Angelis Functional Response and Strong Allee Effect, Math. Comput. Simulation 97, 123-146, 2014.

[33] S.M Rana and U. Kulsum, Bifurcation analysis and chaos control in a discrete-time predator-prey system of Leslie type with simplified Holling type IV functional response, Discrete Dyn. Nat. Soc. 2017, Art. No. 9705985, 2017.

[34] H. Singh, J. Dhar and H.S. Bhatti, Discrete-time bifurcation behavior of a preypredator system with generalized predator, Adv. Difference Equ. 2015, Art. No. 206, 2015.

[35] G. Sucu, Bir Ayrık Zamanlı Av-Avcı Modelinin Kararlılık ve Çatallanma, TOBB Ekonomi ve Teknoloji Üniversitesi Fen Bilimler Enstitüsü, Yüksek lisans Tezi, Ankara, 2016.

[36] S. Wiggins, Introduction to Applied Nonlinear Dynamical System and Chaos, Springer-Verlag, New York, NY, USA, 2003.

[37] L. Zhang and L. Zou, Bifurcations and control in a discrete predator-prey model with strong Allee effect, Int. J. Bifur. Chaos, 28 (5), 1850062, 2018.

[38] J. Zhang, T. Deng, Y. Chu, S. Qin, W. Du, and H. Luo, Stability and bifurcation analysis of a discrete predator-prey model with Holling type III functional response, J. Nonlinear Sci. Appl. 9, 6228-6243, 2016.

[39] S. Zhou, Y. Liu and G. Wang, The stability of predator-prey systems subject to the Allee effects, Theoret. Population Biol. 67, 23-31, 2005. 